\title{
Mentors' competence in mentoring culturally and linguistically diverse nursing students during clinical placement
}

\begin{tabular}{|r|l|}
\hline Journal: & Journal of Advanced Nursing \\
\hline Manuscript ID & JAN-2017-0155.R1 \\
\hline Manuscript Type: & Original Research: Empirical research - quantitative \\
\hline Keywords: & $\begin{array}{l}\text { Clinical Placements, Mentors, Clinical Guidelines, Clinical Supervision, } \\
\text { Cultural Issues, Nurse Education }\end{array}$ \\
\hline Category: & Nursing \\
\hline \multicolumn{2}{|c}{} \\
\hline
\end{tabular}

SCHOLARONE ${ }^{m}$

Manuscripts 


\section{Impact Statement}

This paper advocates for the need to further develop and strengthen nursing education on a global level. Educational and healthcare organizations should work together to ensure that the necessary support structures are in place for future healthcare professionals with diverse backgrounds in order to enable optimal learning outcomes within clinical learning environments. This paper increases knowledge base on mentors' competence in mentoring culturally and linguistically diverse nursing students. This knowledge can be used in the development of strategies to enhance mentors' competence in mentoring, which can ultimately promote a clinical learning environment in which students can provide safe, patient-centered and culturally competent care to the individual patient. 


\section{Abstract}

\section{Aims}

To describe mentors' competence in mentoring culturally and linguistically diverse nursing students during clinical placement and identify the factors that affect mentoring.

\section{Background}

Healthcare education is confronted by several challenges in a time characterized by globalization and increasing international migration. Nursing students from diverse backgrounds continue to experience difficulties during clinical placement. Students can overcome these difficulties and assume responsibility for their learning when mentored by supportive and competent mentors.

\section{Design}

A cross-sectional, descriptive explorative study design was used.

\section{Methods}

Data were collected during spring 2016 through a survey sent to mentors $(n=3355)$ employed at five university hospitals in Finland. Mentors' competence in mentoring culturally and linguistically diverse nursing students was measured with the self-assessment Mentors' Competence Instrument and the Cultural and Linguistic Diversity in Mentoring scale. The analysis included descriptive statistics, nonparametric tests and binary logistic regression analysis.

\section{Results \\ Mentors with experience mentoring nursing students from diverse backgrounds rated their overall competence in mentoring as good. However, the results show continued challenges related to competence in linguistic diversity in mentoring. Seven factors that affect mentors' competence in linguistic diversity were identified. Despite high evaluations by mentors of competence related to cultural diversity in mentoring, there are still opportunities for improvement in this area.}

\section{Conclusion}

Innovative and effective strategies are needed to develop mentors' competence in mentoring culturally and linguistically diverse nursing students. Educational and healthcare organizations should strive to enhance collaboration and increase the competence of both mentors and nursing students to work in increasingly diverse healthcare environments.

Keywords: clinical placement, competency, culturally and linguistically diverse, mentor, mentoring, nursing, students 


\section{SUMMARY STATEMENT}

\section{Why is this research needed?}

- Several studies show that nursing students from culturally and linguistically diverse backgrounds continue to experience the greatest challenges during completion of their clinical placement.

- The mentor is an important source of support and has a significant role in the facilitation of the individual nursing student's learning process and development of the student's professional identity.

- The factors that affect mentors' competence in mentoring culturally and linguistically diverse students have not been previously identified.

\section{What are the key findings?}

- Mentors rated their competence in mentoring culturally and linguistically diverse nursing students positively with the exception of competence in linguistic diversity in mentoring.

- Mentors' competence in linguistic diversity was affected, among other matters, by English language proficiency, experience living or working abroad and frequency of mentoring exchange students.

- Despite mentors' high overall evaluation of their competence in cultural diversity, there is a need to reduce stereotypes and to increase mentors' knowledge of students' cultural background.

\section{How should the findings be used to influence policy/practice/research/education?}

- Educational and healthcare organizations should strive to address the many challenges that culturally and linguistically diverse nursing students and their mentors face in clinical placement.

- Effective support structures need to be in place for both mentors and nursing students in order to promote a clinical learning environment in which students can provide safe and culturally competent care to the individual patient.

- Evidence-based educational programs are needed to enhance mentors' competence in mentoring nursing students from diverse backgrounds. 


\section{INTRODUCTION}

Healthcare systems are characterized by ever increasing workforce diversity as a result of trends in both nurse migration and international migration in general (Bhopal 2014). Diversity in the workforce affects healthcare practices and health systems around the globe (Freeman et al. 2012). Migration increases the need to redirect healthcare education on a global scale (Koehn 2006) and to strengthen the international dimension to nursing education (Levi 2009) and continuing nursing education. In recent years, there has been a steady rise in the amount of nurses with a foreign background in Finland. Despite this increase, these nurses account for a relatively small proportion of the nursing workforce with about 3 percent of practicing nurses being of foreign origin and 1.5 percent of practicing nurses being foreign nationals (National Institute for Health and Welfare 2015). In Finland and elsewhere, it is imperative that educational and healthcare organizations strive to promptly address possible challenges that emerge related to increasing diversity, and implement strategies to develop current and future healthcare professionals' competence to work in diverse healthcare settings.

The global strive to ensure equitable access to health workers and to strengthen the sustainability of health systems is ongoing (WHO 2016). In the WHO European Region, there is a goal to scale up and transform education and training for nurses, the group of healthcare professionals that collectively forms the largest component of the health workforce (WHO 2015). The European Union sets standards for nursing education, for instance, through stating that the duration of clinical training should account for at least one half of the minimum duration of the nursing program. In addition, clinical training must be completed under the supervision of qualified nursing staff (Directive 2013/55/EU of the European Parliament and of the Council). 
Several studies show that nursing students from culturally and linguistically diverse (CALD) backgrounds experience the greatest challenges during completion of their clinical placement (Edgecombe et al. 2013, O'Reilly \& Milner 2015, Authors names blinded, Authors names blinded). Nursing students should all receive equal opportunities in education regardless of their backgrounds. Equal opportunity for all is seen as the foundation of populations' welfare (Ministry of Education and Culture 2012), however, students from immigrant backgrounds are underrepresented in higher education, often due to a lack of proficiency in the local language (Authors names blinded). Mentors' competence in mentoring CALD nursing students during their clinical placement should be studied further because mentors play an important role in students' experiences and learning outcomes (Authors names blinded), and because the clinical placement, where the most challenges occur (Pitkäjärvi et al. 2012a), accounts for such a large proportion of nursing education. The factors that affect mentoring need to be identified in order to further develop clinical learning environments to support the learning of CALD nursing students.

\section{BACKGROUND}

Clinical placements are an important part of nursing education and have a significant impact on the professional development of nursing students (Carlson et al. 2009, Arieli 2013, Authors names blinded). During the mentoring of a student in clinical placement, a healthcare professional supports the student to achieve his/her goals and to grow and develop professionally (Carlson et al. 2009, Barker \& Pittman 2010). Mentoring happens in a goal-oriented relationship within a predetermined timeframe, in which the mentor presents the reality of healthcare practice to the student (Barker \& Pittman 2010). In this study, the 'mentor' refers to a healthcare professional who teaches and supports the nursing student during their clinical placement. Mentors who display competence in mentoring have the necessary knowledge and skills to identify and understand the role and tasks of the mentor, motivate and support 
the learning process of the student, promote reflective practices and provide constructive feedback and evaluation (Karjalainen et al. 2015).

The concept of 'culturally and linguistically diverse' (CALD) is used in this study to refer to nursing student whose background differs from the mainstream culture and language (Robinson \& Clardy 2011). In the context of this study, having a CALD background means that the student has a native language, cultural background or heritage differing from that of the country he/she studies in. O'Reilly and Milner (2015) found that both mentors and students experienced that the students' cultural and linguistic background had an impact on clinical placements. Mentors and students reported that culture differences have an impact on learning and teaching styles with, for instance, difficulties in feedback, self-directed learning and reflective practices (O’Reilly \& Milner 2015). In addition, several challenges exist when language barriers hinder communication between the mentor and student. In positive clinical learning environments the student's lack of language proficiency does not hinder learning (Myhre 2011, Pitkäjärvi et al. 2012b), different languages are used flexibly in different situation (Mattila et al. 2010) and the student receives the necessary support from the mentor for enabling communication (Authors names blinded).

The importance of positive clinical learning environments cannot be emphasized enough as it has been shown in various studies to have a significant impact on the experiences that CALD nursing students have during their clinical placement (Mattila et al. 2010, Myhre 2011, Pitkäjärvi et al. 2011, Pitkäjärvi et al. 2012b, Edgecombe et al. 2013, Authors names blinded). The role of a mentor for culturally and linguistically diverse nursing students is a challenging one, and several studies prove the need to develop mentors' competence in mentoring through educational programs especially in regards to competencies 
in cultural and linguistic diversity (San Miguel et al. 2006, Mattila et al. 2010, Jeong et al. 2011, Myhre 2011, Pitkäjärvi et al. 2011, Pitkäjärvi et al. 2012a, Pitkäjärvi et al. 2012b, Arieli 2013, Edgecombe et al. 2013, Sedgwick et al. 2014, Authors names blinded, Authors names blinded).

\section{THE STUDY}

Aim

The aim of the study was to describe mentors' competence in mentoring culturally and linguistically diverse nursing students during clinical placement and identify the factors that affect mentoring. The research questions were (1) What kind of competencies do mentors have in mentoring CALD nursing students as evaluated by the mentors' themselves? and (2) Which factors affect the mentoring of CALD nursing students in the clinical learning environment?

\section{Design}

A cross-sectional, descriptive explorative study design was used.

\section{Participants}

The study population consisted of mentors within all five university hospitals in Finland selected with stratified sampling technique (Grove et al. 2013). The university hospitals are located in five major cities in Finland, and serve the entire population of Finland. The stratified sampling technique was used by counting the total amount of registered nurses $(N=13$ 342) working in all five university hospitals. The stratified groups were divided into five groups according to the proportional amount of registered nurses representing the five different hospitals. The participants were chosen randomly from each of the five groups with a total of 3355 nurses receiving an invitation to participate in the study. The inclusion criteria set for the participation in this study were: (1) be currently employed at one of the university hospitals in any professional registered nursing related role on any unit (2) have experience in mentoring students who study in registered nursing degree related programmes; (3) have the skills to read and 
understand the Finnish language. A study sample was drawn from the participants who answered the survey, which consisted of mentors who met the above inclusion criteria, in addition to having prior experience mentoring either nursing students in exchange programmes, CALD nursing students in an English language-taught degree programme or CALD nursing students in a Finnish degree programme.

\author{
Data Collection \\ Data were collected during spring 2016 using a survey accompanied by a covering letter that was sent to \\ the mentors 'work email addresses at all of the five university hospitals in Finland. All study participants \\ received reminder emails two weeks following the initial survey; two reminder emails were sent at three \\ participating hospitals and one reminder email at the remaining two hospitals. There was a total of $n=$ \\ 576 respondents to the survey. The overall response rate was $17.2 \%$. The data sample used in this study \\ consisted of all respondents to the questionnaire who had experience in mentoring CALD nursing \\ students $(n=323,56.1 \%)$.
}

\title{
Instruments
}

The self-assessment instrument used in this cross-sectional study consisted of a Mentors' Competence Instrument (MCI) (Karjalainen et al. 2015, Kälkäjä et al. 2016) and newly developed self-assessment Cultural and Linguistic Diversity in Mentoring scale (CALD+Ms) with background information. The Likert scale with four response categories (1- fully disagree; 2- disagree to some extent; 3- agree to some extent; and 4- fully agree) was utilized.

\section{The Mentors`Competence Instrument}

The MCI consisted of nine sub-dimensions with 55 items. The sub-dimensions are comprised of: (1) mentor characteristics; (2) identifying the student's level of competence; (3) motivation of the mentor; (4) motivating the student; (5) supporting the learning process of the student; (6) goal orientation in 
mentoring; (7) reflection during mentoring; (8) student-centred feedback and evaluation; (9) constructive feedback and evaluation.

\section{Cultural and Linguistic Diversity in Mentoring Scale}

The newly developed CALD+Ms scale consisted of two sub-dimensions: (1) cultural diversity in mentoring (8 items) and (2) linguistic diversity in mentoring (6 items). The scale was developed based on two systematic reviews, which relate to the experiences that CALD nursing students (Authors names blinded) and their mentors (Authors names blinded) encounter in the clinical learning environment. Eight experts in clinical mentorship evaluated the face and content validity of the scale with the method of Content Validity Index, and the result was excellent (CVI = .94) (Polit et al. 2007). The original amount of items (16) were minimized by two items (14), and clarification was performed on all aspects of the items. The CALD+Ms was pilot tested by 35 mentors in order to evaluate the practicality and interpretation of the items (Sue \& Ritter 2007).

Prior to construct validity testing of the CALD+Ms scale, the correlation between the variables were examined through Spearman`s correlation coefficient. The range for inter item correlation was set at between $r=.30$ and .70 (Pett et al. 2003, Polit \& Beck 2012). The variables which did not correlate with other variables or overload with other items were removed (Munro 2005). Three items were removed from the cultural diversity in mentoring sub-dimension and three items were removed from the linguistic diversity in mentoring sub-dimension. Exploratory factor analysis was conducted to test the construct validity. The Bartlett Test for Sphericity $751.941(d f=28, P<0.01)$ and the Kaiser-Meyer-Olkin test .75 were performed; the data was found fit for exploratory factor analysis (Munro 2005). The number of factors were estimated with eigenvalues set to be greater than 1 and through examination of scree plot (Yong \& Pearce 2016). Principle axis factoring was used for extraction (DeVellis 2012). The first factor 
the cultural diversity in mentoring had an eigenvalue of 3.09 and a total item variance explained by the factor of 38.7\%. The second factor the linguistic diversity in mentoring had an eigenvalue of 1.59 and a total item variance explained by the factor of $19.8 \%$. The factor loading cutoff was set at an absolute value of 0.40 (Grove et al. 2013). Promax rotation was used to rotate the factor loading matrix (Williams et al. 2012) (Table 1).

\section{Ethical Considerations}

The study was approved through written consent from all of the participating university hospitals according to each hospital's research approval protocol. This cross-sectional study did not require formal approval from an ethics committee (Medical Research Act 2010/794). In addition, written agreement was received for the use of the instruments in this study. Participation in the study was voluntary, and completing the survey was taken as agreement to participate in the study. Participants were informed in the covering letter of the aim of the study as well as of their right to withdraw from the study at any time. There was no physical and psychological harm caused to participants of the study. The results are presented in a confidential manner without possibility of participant identification (Personal Data Act 523/1999). The data were kept in a safe place accessible only to the researchers undertaking the research project. The data will be completely destroyed after there is no use for the data in the research project. (RCR 2012).

\footnotetext{
Data Analysis

This cross-sectional, descriptive explorative study strived to describe and identify factors affecting outcome variables (Polit \& Beck 2012). IBM SPSS (V23.0) was used in the analysis of the data. The analysis included descriptive statistics (frequencies, percentages, means, standard deviation), Spearman's rank order correlation $(P)$ tests, nonparametric tests and binary logistic regression analysis. The significance level for all statistical tests was set at $P<0.05$ (Munro 2005).
} 
Prior to binary logistic regression analysis, the Spearman's rank correlation coefficients for the background variables were examined in the form of a correlation matrix. Logistic regression analysis was used to analyse the relationship between the multiple independent variables and dependent variable in order to yield a predictive model (Munro 2005). The four-level Likert scale was minimized into dichotomous response categories by dividing the Likert scale into half according to the meaning of the items in the scale: $(0)$ poor competence (1-2.49) and (1) good competence (2.50-4). The odds ratio $(O R)$ with $95 \%$ confidence interval $(C I)$ were calculated for each factor. Initially, a backward elimination method with theoretical guidance on the subject matter (Authors names blinded, Authors names blinded ) was used during the finding of the most fitting logistic regression model. Then the model was retested by the manually build no-stepwise method while testing each independent variable individually and combining the variables into the model presented in this study. The independent variables were the same in the retested method. The goodness of fit statistic (-2 LL) was tested by the Hosmer-Lemeshow test, which deemed a nonsignificant result that proves the model fits (Munro 2005). The effect size was counted in odds ratio $(O R)$ estimation with interpretation values small $(O R=1.5)$, moderate $(O R=2.5)$, large $(O R=4)$ and very large $(O R=10)($ Lakens 2013).

\section{Validity and reliability of the study \\ The validity of the newly developed CALD+Ms scale was ensured by conducting face and content validity, construct validity, and reliability. The development of the scale began with a structured theoretical framework based on the latest evidence-based knowledge (DeVellis 2012) found in two systematic reviews (Authors names blinded, Authors names blinded). Face and content validity was completed through careful selection of experts in the research area and through completion of the Content Validity Index (CVI) method (Cook \& Beckman 2006). The construct validity was ensured by}


completing exploratory factor analysis with principle axis factoring with oblique rotation (Miettunen 2004, Williams et al. 2012). The pilot study conducted prior to main data collection ensured avoidance of technical challenges and prevented possible bias due to misunderstanding of the items. The Cronbach's alpha for the CALD+Ms scale varied between 0.75 and 0.77 , which can be considered adequate for a newly developed scale (Rattray \& Jones 2007). In this study, the internal consistency reliability of the previously used MCI (Karjalainen et al. 2015, Kälkäjä et al. 2016) instrument was tested, and the results varied between 0.75 and 0.92 .

\section{RESULTS}

\section{Mentors' background}

The background factors of the mentors with experience mentoring CALD nursing students can be seen in Table 2. Study participants were mostly female (86\%); aged 23-66 $(M=41.72, S D 10.91)$; of Finnish decent (97.8\%) with Finnish as their native language (96.5\%); held a university of applied sciences level degree (92\%); with under 20 years of work experience (69.6\%); and current work unit either an outpatient clinic (34.7\%) or inpatient unit (53.6\%). The majority of participants had mentored students as either a named mentor $(40.2 \%)$ or mentor $(43.3 \%)$; and mentored students within the past month (71.8\%). Over half of the mentors (57.9\%) had not participated in additional mentoring training. The majority of mentors $(88.3 \%)$ rated their overall mentoring competence as 'good' or excellent.

About $70 \%$ of mentors had not previously lived or worked abroad. The study participants reported experience in mentoring CALD nursing students from exchange programmes (68.4\%), English language-taught degree programmes (67.8\%) and Finnish degree programmes (61.6\%). The majority of mentors rated their English language skills as advanced (80.5\%). Half of the mentors (50.5\%) reported that they require sufficient Finnish or Swedish language skills from CALD students. More mentors 
reported insufficient knowledge of the CALD student's cultural background (52.6\%) than sufficient, and the majority of mentors ensured that CALD and native students work together $(77.1 \%)$. Over half of the mentors (77.4\%) reported that they spend time discussing cultural differences with CALD students. The majority of mentors (76.2\%) agreed that they have received additional support from their colleagues in mentoring CALD nursing students with less than half of the mentors $(49.2 \%)$ stating that they need more support from colleagues.

\section{Mentors' competence in mentoring CALD nursing students}

Mentors with experience in mentoring CALD nursing students evaluated their mentoring competence positively. Results from the MCI displayed mean values, which varied between 3.18 and 3.72 on the four-level Likert scale (Table 3). The sum variable reflection during mentoring received the highest evaluation with a mean of 3.72 (SD 0.37), which was followed by identifying the student's level of competence $(M=3.68, S D 0.44)$. The sum variables mentor characteristics $(M=3.57, S D 0.40)$ and supporting the learning process of the student $(M=3.51, S D=0.37)$ received similar mean values. The following four sum variables also displayed similar mean values: motivation of the mentor $(M=3.42$, $S D$ 0.54), motivating the student $(M=3.49, S D 0.46)$, goal orientation in mentoring $(M=3.45, S D 0.51)$ and constructive feedback and evaluation $(M=3.48, S D$ 0.42). The student-centred feedback and evaluation sum variable received the lowest evaluation $(M=3.18, S D$ 0.56). Mentors evaluated competence in cultural and linguistic diversity in mentoring mainly positively (Table 1). The mean values varied between 3.52 (SD 0.43 ) for cultural diversity in mentoring and 2.58 (SD 0.72 ) for linguistic diversity in mentoring on the four-level Likert scale. 


\section{Factors that affect mentors' competencies}

Factors that affect mentors' linguistic diversity competency in mentoring CALD nursing students in clinical placement were identified in this study (Table 4). All of the sub-dimensions of MCI and CALD+Ms with the exception of linguistic diversity in mentoring did not fit binary logistic regression analysis because the majority of the mentors evaluated their competence on a highly agreeable level and the comparison groups remained too small in size.

The factors that were identified for the best fit of the model were mentors' English language proficiency; experience living and/or working abroad; frequency of mentoring exchange students; sufficient knowledge on the students' cultural background; time spent discussing cultural differences with students; the integration of CALD students together with native students; and the need to receive additional support from colleagues. The outcome variable was explained by factors $37.8 \%$ with scoring significant values showing fitting of the model (Table 4).

Mentors who reported basic proficiency in the English language had a decreased rating of their competence in linguistic diversity in mentoring $(O R=0.20,95 \% C I=0.10-0.42, P<0.01)$ compared to mentors who reported advanced competence in the English language. Mentors who had experience living or working abroad had a higher rate of competence in linguistic diversity $(O R=1.82,95 \% C I=$ 1.00-3.29, $P=0.05$ ) than mentors who had no experience living or working abroad. The frequency of mentoring exchange students increased competence in linguistic diversity $(\mathrm{OR}=3.46,95 \% C I=1.60-$ $7.48, P=0.02)$. Mentors who had sufficient knowledge of the student's cultural background $(O R=$ $1.89,95 \% C I=1.10-3.25, P=0.02)$, spent time discussing cultural difference with students $(O R=2.14$, $95 \% C I=1.08-4.27, P=0.03)$, and ensured that CALD and native students work together $(O R=2.36$, $95 \% C I=1.21-4.62, P<0.01)$ had a higher rate of competence in linguistic diversity. In the end, 
mentors with a higher rating of competence in linguistic diversity needed less support from colleagues in mentoring CALD students $(O R=0.35,95 \% C I=0.21-0.60, P<0.01)$. The effect size of the binary logistic regression analysis model varied from moderate $(O R=1.82, O R=1.89, O R=2.14, O R=2.36$, $O R=2.86, O R=3.48)$ to large $(O R=5.00)$.

\section{DISCUSSION}

Mentors with experience mentoring cultural and linguistic diverse nursing students during clinical placement evaluated their overall mentoring competence highly. Competence in linguistic diversity in mentoring, which received the poorest evaluation by mentors in this study, was an exception. Previous studies also show that challenges related to communication create obstacles in the clinical learning environment for CALD nursing students (Authors names blinded, Authors names blinded) and their mentors (Authors names blinded). Effective communication plays a central role in healthcare (San Miguel et al. 2006). It is crucial in enabling positive outcomes and experiences during clinical placement (Authors names blinded) and requires competence and involvement from both mentors and students (Pitkäjärvi et al. 2012a).

Due to patient safety implications, nurses are required to have sufficient knowledge of the language necessary for practicing nursing (Directive 2013/55/EU of the European Parliament and of the Council). Increased diversity in health systems and in the healthcare workforce has stimulated initiatives for educational institutions to put higher emphasis on internationalization and on the language proficiency of professionals (Ministry of Education 2009). In Finland nurses who have graduated prior to such initiatives have had less obligatory education or training in English, which may emphasize the need for healthcare organizations to provide additional education and support to staff members. Educational institutions also play an important role in providing the necessary support structures that mentors need to 
ensure quality mentoring (Barnett et al. 2010) and to overcome possible language barriers or other challenges.

Mentors in this study who reported basic proficiency in English rated their competence in linguistic diversity poorly compared to those with advanced proficiency in English. In this study, a higher level of competence in linguistic diversity affected good mentoring practices, which in turn enhances students' learning (Pinto Zipp \& Kolber 2014). These mentors had sufficient knowledge of the students' cultural background, invested in spending time to discuss cultural differences with students and ensured that CALD nursing students worked together with native students. Previously, mentors expressed mentoring CALD nursing students in a foreign language as stressful, demanding and exhausting (Pitkäjärvi et al. 2011), indicating a need for additional support from educational training or peer support from colleagues. In this study, the majority of mentors reported receiving additional support from colleagues during the mentoring of CALD nursing students. Half of the mentors agreed that they need more support from colleagues. However, mentors with higher competence in linguistic diversity in mentoring required less support from their colleagues.

Studies show that communication related challenges increase in interactions between patients, healthcare staff and students especially in situations where the student lacks knowledge of the local language (San Miguel et al. 2006, San Miguel \& Rogan 2009, Mattila et al. 2010, Jeong et al. 2011, Pitkäjärvi et al. 2011, Pitkäjärvi et al. 2012a, Pitkäjärvi et al. 2012b, Arieli 2013, Sedgwick et al. 2014). Students’ selfconfidence increased when they were able to communicate despite language barriers, and these experiences helped them grow professionally (Myhre 2011). According to an evaluation on entrance examination in Finland of CALD nursing students completing their degree in English language-taught 
degree programme, two out of ten universities of applied sciences set requirements for Finnish language proficiency prior to acceptance to the degree programme (Study-info 2017). CALD nursing students are required to complete their clinical placements in the local language, despite the nursing degree programme being offered in English (Authors names blinded). Half of the mentors in this study had expectations for CALD nursing students to be able to use the local language during completion of their clinical placement. Students' knowledge of the local language is an important factor influencing the outcomes of a successful clinical learning environment and mentoring (Authors names blinded). Educational institutions that educate CALD nursing students should consider students' language competence, and offer clinical language courses to students prior to clinical placements. This enables students' language competence to meet the required level needed to complete their clinical placements. In a previous study, approximately half of CALD nursing students evaluated their local language skills as beginner level upon entry to their clinical placement with poor knowledge of clinical vocabulary in the local language (Authors names blinded).

Experience living or working abroad had a positive effect on mentors' competence in linguistic diversity, although the majority of mentors reported having no previous experience living or working abroad. In Europe, educational institutions continue to strengthen high-quality exchange programmes for incoming and outgoing students (Hvalic-Touzery et al. 2017). Experience abroad is seen as an asset for future employers. Studies show that, despite challenges, these experiences increase students' cultural awareness and language proficiency along with promote their professional and personal growth (Mattila et al. 2010, Myhre 2011, Pitkäjärvi et al. 2011, Pitkäjärvi et al. 2012b, Edgecombe et al. 2013). International experience can also be gained in one's own organization through, for example, the mentoring of CALD nursing students in exchange programmes, which was proven in this study to have 
a positive effect on mentors' competence in linguistic diversity in mentoring. Employers should continue to develop strategies for the continued development of staff' language proficiency and encourage staff to gain international experience in healthcare.

The item that received the lowest evaluation in this study was the cultural diversity in mentoring subdimension concerning mentors' evaluation of not stereotyping culturally diverse students. Positive learning environments help to overcome barriers related to cultural diversity (Pitkäjärvi 2012c) and minimize ethnic stereotyping of students (Arieli 2013). Efforts to identify and decrease stereotyping of students create the conditions for successful mentoring (Edgecombe et al. 2013). Several studies show that students continue to encounter stereotypes, discrimination and racism from staff members and patients (Mattila et al. 2010, Jeong et al. 2011, Arieli 2013, Sedgwick et al. 2014). This issue should be addressed for discrimination has serious negative consequences on the experiences and learning outcomes of students; it causes intimidation and frustration (Mattila et al. 2010, Jeong et al. 2011, Arieli 2013). An essential part of improvement and development of clinical placements is the building of safe clinical environments for CALD nursing students and their mentors. Students and their mentors could benefit from further education regarding enhancement of competencies in cultural and linguistic diversity in order to ensure safety in the clinical learning environment.

\section{Study Limitations}

This cross-sectional study was characterized by a low overall response rate $(17.2 \%)$, which potentially causes bias in the interpretation of the results. Low online survey response rates are an increasing challenge faced by researchers (Olson 2014). The data in this study was collected in close collaboration between the researchers and the healthcare organizations with sufficient planning and resources. Despite the low response rate, the sample size represented the required population with stratified sampling technique and effect size scores that show an efficient sample size (Lakens 2013). 
The instruments used in this study were tested in the Finnish context, therefore careful utilization of results in other contexts is required (Flanders et al. 2016). The evaluations by mentors in this study were fairly high with possible over evaluated outcomes in some areas. The outcomes brought hindrances for the utilization of binary logistic regression with groups of mentors with lower competence being too small to use in the statistical analysis. Linguistic diversity in mentoring was the only normally distributed sum variable for which logistic regression analysis could be performed.

\section{CONCLUSION}

This cross-sectional study builds on previous research and increases knowledge base on mentors' competence in mentoring culturally and linguistically diverse nursing students through the identification of background factors that affect mentoring. This study offers several opportunities to further expand research on mentors' competence in mentoring. Experimental studies could be designed to measure the effects of educational interventions on mentors' competence. There is a need for the development of evidence-based educational programmes that enhance mentors' competence in mentoring CALD nursing students. In addition, this study can be extended in the search for further knowledge of mentors' competence in various countries. The validated instruments in this study could be utilized in crosscultural studies, which could open the possibility for comparable studies on clinical practices in different countries.

In this study, there was variation in mentors' requirements towards students' knowledge of the local language. Variation in expectations stimulates the need for consistency in language requirements and assessment of the students' language skills prior to clinical placements. Students need a comprehensive orientation into the clinical placement in order to enhance familiarization with the clinical environment and the mentor. The mentor also needs sufficient knowledge of the students' background, and needs 
time to reflect upon possible cultural differences with the student. The strengthening of collaboration between educational institutions and healthcare organizations may improve the support structures provided to mentors, and in the end, enhance relations between mentors and students.

There continues to be a need for innovative and effective strategies aimed to ensure high-quality mentoring and to develop the understanding, acceptance and appreciation for nursing students with diverse backgrounds in the clinical setting. The experiences and knowledge that CALD nursing students bring to clinical learning environments should be viewed as an asset and strategic resource for healthcare organizations, instead of as solely and endangerment of patient safety or cause of other serious problems. Surely a student-centred approach where the individual needs of the student are recognized could facilitate the completion of clinical placements in a positive learning environment, where the student can provide patient-centred and culturally competent care without compromising the safety of the patient.

\section{REFERENCES}

Arieli D. (2013) Emotional work and diversity in clinical placements of nursing students. Journal of Nursing Scholarship 45(2), 192-201. doi: 10.1111/jnu.12020

Barker E.R. \& Pittman O. (2010) Becoming a super preceptor: A practical guide to preceptorship in today's clinical climate. Journal of the American Academy of Nurse Practitioners 22(3), 144-149. doi: 10.1111/j.1745-7599.2009.00487.x

Barnett T., Cross M., Shahwan-Akl L. \& Jacob E. (2010) The evaluation of a successful collaborative education model to expand student clinical placements. Nurse Education in Practice 10(1), 71-21. doi: http://dx.doi.org.pc124152.oulu.fi:8080/10.1016/j.nepr.2009.01.018

Bhopal R.S. (2014) Migration, Ethnicity, Race and Health in Multicultural Societies. 2nd edn. Oxford University Press, Oxford.

Carlson E., Wann-Hansson C. \& Pilhammar E. (2009) Teaching during clinical practice: Strategies and techniques used by preceptors in nursing education. Nurse Education Today 29(5), 522-526. doi: http://dx.doi.org.pc124152.oulu.fi:8080/10.1016/j.nedt.2008.11.012

Cook D.A. \& Beckman T.J. (2006) Current concepts in validity and reliability for psychometric instruments: Theory and application. American Journal of Medicine 119, 166.

DeVellis R. (2012) Scale Development. Theory and Applications. 3rd edn. Sage Publications, Newbury Park NT.

Directive 2013/55/EU of the European Parliament and of the Council. Official Journal of the European Union No L 354/132. Available at: http://data.europa.eu/eli/dir/2013/55/oj (accessed 11 January 2017). 
Edgecombe K., Jennings M. \& Bowden M. (2013) International nursing students and what impacts their clinical learning: Literature review. Nurse Education Today 33(2), 138-142. doi: http://dx.doi.org.pc124152.oulu.fi:8080/10.1016/j.nedt.2012.07.015

Flanders W.D., Klein M. \& Mirabelli M.C. (2016) Conditions for valid estimation of causal effects on prevalence in cross-sectional and other studies. Annals of Epidemiology 26(6): 389-394. doi: http://dx.doi.org.pc124152.oulu.fi:8080/10.1016/j.annepidem.2016.04.010

Freeman M., Baumann A., Blythe J., Fisher A. \& Akhtar-Danesh N. (2012) Migration: A concept analysis from a nursing perspective. Journal of Advanced Nursing 68(5), 1176-1185. doi: $10.1111 / \mathrm{j} .1365-2648.2011 .05858 . x$

Grove S.K., Burns N. \& Gray J.R. (2013) The Practice of Nursing Research: Appraisal, Synthesis, and Generation of Evidence. 7th edn. Elsevier, St. Louis, Missouri.

Hvalič-Touzery S., Hopia H., Sihvonen S., Diwan S., Sen S. \& Skela-Savič B. (2017) Perspectives on enhancing international practical training of students in health and social care study programs - A qualitative descriptive case study. Nurse Education Today 48, 40-47.

Jeong S.Y.-S., Hickey N., Levett-Jones T., Pitt V., Hoffman K., Norton C.A. \& Ohr S.O. (2011) Understanding and enhancing the learning experiences of culturally and linguistically diverse nursing students in an Australian bachelor of nursing program. Nurse Education Today 31(3), 238-244. doi: http://dx.doi.org.pc124152.oulu.fi:8080/10.1016/j.nedt.2010.10.016

Karjalainen T., Ruotsalainen H., Sivonen P., Tuomikoski A.-M., Huhtala S. \& Kääriäinen M. (2015) [Mentors' assessment of their competence in mentoring] (in Finnish). Hoitotiede 27(3), 183-198.

Koehn P.H. (2006) Globalization, migration health, and educational preparation for transnational medical encounters. Globalization and Health 2(2), 1-16. doi: 10.1186/1744-8603-2-2

Kälkäjä M., Ruotsalainen H., Sivonen P., Tuomikoski A.-M., Vehkaperä A. \& Kääriäinen M. (2016) [Student counselling practices, resources and mentors in the health industry - A student mentor's view] (in Finnish). Hoitotiede 28(3), 229-242.

Lakens D. (2013) Calculating and reporting effect sizes to facilitate cumulative science: A practical primer for t-tests and ANOVAs. Frontiers in Psychology 4:863. doi: 10.3389/fpsyg.2013.00863

Levi A. (2009) The ethics of nursing student international clinical experiences. Journal of Obstetric, Gynecologic \& Neonatal Nursing 38(1), 94-99. doi: 10.1111/j.15526909.2008.00314.x

Mattila L.-R., Pitkäjärvi M. \& Eriksson E. (2010) International student nurses' experiences of clinical practice in the Finnish health care system. Nurse Education in Practice 10(3), 153-157. doi: http://dx.doi.org.pc124152.oulu.fi:8080/10.1016/j.nepr.2009.05.009

Medical Research Act. (2010/794) Ministry of Social Affairs and Health, Finland. Available at: http://www.finlex.fi/fi/laki/kaannokset/1999/en19990488 (accessed 11 January 2017).

Miettunen J. (2004) Statistical Methods in Psychiatric Research, with Special Reference on Factor Analysis. Academic Dissertation. Oulu, Acta Universitatis Ouluensis.

Authors names blinded (2016a) Culturally and linguistically diverse healthcare students' experiences of learning in a clinical environment: A systematic review of qualitative studies. International Journal of Nursing Studies 54, 173-187. doi: http://dx.doi.org/10.1016/j.ijnurstu.2015.06.004

Authors names blinded (2016b) Mentor experiences of international healthcare students' learning in a clinical environment: A systematic review. Nurse Education Today 40, 87-94. doi: http://dx.doi.org.pc124152.oulu.fi:8080/10.1016/j.nedt.2016.02.013

Authors names blinded (2017) Clinical learning environment and supervision of international nursing students: A cross-sectional study. Nurse Education Today. 
Ministry of Education. (2009) Strategy for the Internationalisation of Higher Education Institutions in Finland 2009-2015. Publication of the Ministry of Education, Finland 2009:23.

Ministry of Education and Culture. (2012) Education and Research 2011-2016. A development plan. Available

at: http://www.minedu.fi/OPM/Julkaisut/2012/Kehittamissuunnitelma.html?lang=fi\&extra_locale=en (accessed 6 January 2017).

Munro B.H. (2005) Statistical Methods for Health Care Research. 5th edn. Lippincott Williams \& Wilkins, Philadelphia.

Myhre K. (2011) Exchange students crossing language boundaries in clinical nursing practice. International Nursing Review 58(4), 428-433. doi: 10.1111/j.1466-7657.2011.00904.x

National Institute for Health and Welfare. 2015. International Mobility of Health Care and Social Welfare Personnel 2012. Statistical report.

Olson C.A. (2014) Survey burden, response rates, and the tragedy of the commons. Journal of Continuing Education in the Health Professions 34(2), 93-95. doi: http://dx.doi.org.pc124152.oulu.fi:8080/10.1002/chp.21238

O'Reilly S.L. \& Milner J. (2015) Supporting culturally and linguistically diverse students during clinical placement: Strategies from both sides of the table. BMC Medical Education 15:175, 1-12. doi: 10.1186/s12909-015-0458-3

Personal Data Act. (523/1999) Ministry of Justice. Finland. Available at: http://www.finlex.fi/en/laki/kaannokset/1999/19990523 (accessed 11 January 2017).

Pett M.A., Lackey N.R. \& Sullivan J.J. (2003) Making Sense of Factor Analysis. The Use of Factor Analysis for Instrument Development in Health Care Research. Sage Publications, Thousand Oaks.

Pinto Zipp G. \& Kolber C. (2014) Identifying teachable moments in the clinical setting and possible barriers. Journal of Allied Health 43(1), 32-37.

Pitkäjärvi M., Eriksson E. \& Kekki P. (2011) Teachers' experiences of English-language-taught degree programs within health care sector of Finnish polytechnics. Nurse Education Today 31(6), 553-557. doi: http://dx.doi.org.pc124152.oulu.fi:8080/10.1016/j.nedt.2010.10.032

Pitkäjärvi M., Eriksson E. \& Pitkälä K (2012a) The diversity issue revisited: International students in clinical environment. International Scholarly Research Notices 2012, 1-6. doi: http://dx.doi.org/10.5402/2012/294138

Pitkäjärvi M., Eriksson E., Kekki P. \& Pitkälä K. (2012b) Culturally diverse nursing students in Finland: Some experiences. International Journal of Nursing Education Scholarship 9(1), 1-16. doi: 10.1515/1548-923X.2356

Pitkäjärvi M.A. (2012c) English-language-taught degree programmes in faculties of healthcare in Finnish Universities of Applied Sciences: Students' and teachers' conceptions of the implementation. Academic Dissertation. University of Helsinki. Faculty of Medicine.

Polit D.F., Beck C.T. \& Owen S.V. (2007) Is the CVI an acceptable indicator of content validity? Appraisal and recommendations. Research in Nursing \& Health 30(4), 459-467.

Polit D.F. \& Beck C.T. (2012) Nursing Research: Generating and Assessing Evidence for Nursing Practice. 9th edn. Lippincott Williams \& Wilkins, Philadelphia.

Rattray J. \& Jones M.C. (2007) Essential elements of questionnaire design and development. Journal of Clinical Nursing 16(2), 234-243. doi: 10.1111/j.1365-2702.2006.01573.x

RCR (2012) Responsible conduct of research and procedures for handling allegations of misconduct in Finland - RCR guidelines. Finnish Advisory Board on Research Integrity. Available at: http://www.tenk.fi/en/resposible-conduct-research-guidelines (accessed 11 January 2017). 
Robinson C.C. \& Clardy P. (2011) It ain't what you say, it's how you say it: Linguistic and cultural diversity in the classroom. Journal of Cultural Diversity 18(3), 101-110.

San Miguel C., Rogan F., Kilstoff K. \& Brown D. (2006) Clinically speaking: A communication skills program for students from non-English speaking backgrounds. Nurse Education in Practice 6(5), 268-274. doi: http://dx.doi.org/10.1016/j.nepr.2006.02.004

San Miguel C. \& Rogan F. (2009) A good beginning: The long-term effects of a clinical communication programme. Contemporary $\quad$ Nurse $33(2), \quad 179-190 . \quad$ doi: http://dx.doi.org.pc124152.oulu.fi:8080/10.5172/conu.2009.33.2.179

Sedgwick M., Oosterbroek T. \& Ponomar V. (2014) "It all depends": How minority nursing students experience belonging during clinical experiences. Nursing Education Perspectives 35(2), 89-93. doi: http://dx.doi.org.pc124152.oulu.fi:8080/10.5480/11-707.1

Study info (2017) Ministry of Education and Culture. Finnish National Board of Education. Finland. Available at: https://studyinfo.fi/wp2/en/ (accessed 20 April 2017).

Ministry of Education and Culture. (2012) Education and Research 2011-2016. A development plan. Available at: http://www.minedu.fi/OPM/Julkaisut/2012/Kehittamissuunnitelma.html?lang=fi\&extra_locale=en (accessed 6 January 2017).

Sue V.M. \& Ritter L.A. (2007) Conducting Online Surveys. SAGE Publications, Los Angeles, USA.

WHO (World Health Organization). (2015) European strategic directions for strengthening nursing and midwifery towards Health 2020 goals. WHO Regional Office for Europe. Available at: http://www.euro.who.int/en/health-topics/Health-systems/nursing-andmidwifery/publications/2015/european-strategic-directions-for-strengthening-nursing-and-midwiferytowards-health-2020-goals (accessed 5 February 2017).

WHO (2016) Global strategy on human resources for health: Workforce 2030. Available at: http://who.int/hrh/resources/pub_globstrathrh-2030/en/ (accessed 5 February 2017).

Williams B, Brown T \& Onsman A (2012) Exploratory factor analysis: A five-step guide for novices. Australasian Journal of Paramedicine 8(3), 1-12.

Yong A.G. \& Pearce S. (2013) A beginner guide to factor analysis: Focusing on exploratory factor analysis. Tutorials in Quantitative Methods for Psychology 9(2), 79-94. 
Table 1. CALD+Ms scale's explorative factor analysis, sub-dimensions and items $(\boldsymbol{n}=\mathbf{3 2 3})$

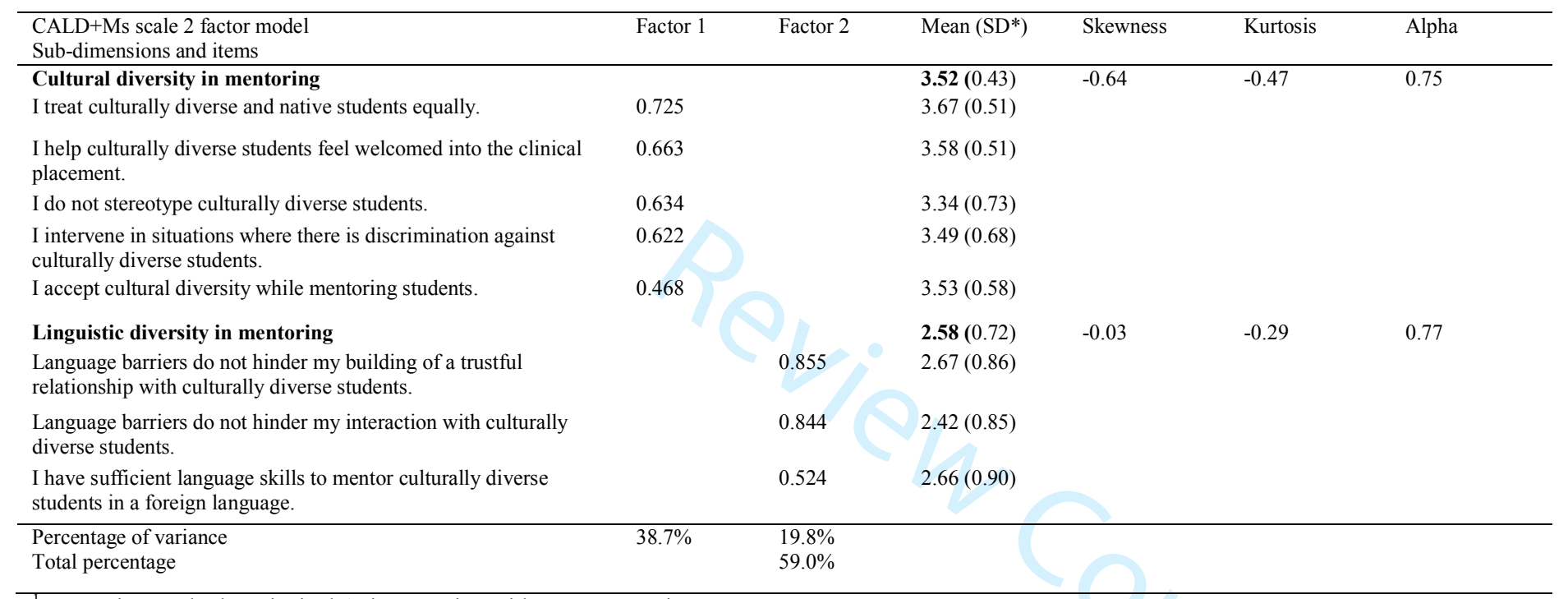

${ }^{1}$ Extraction methods: Principal Axis Factoring with Promax rotation

Note. $* \mathrm{SD}=$ standard deviation 
Table 2. Background factors of mentors $(n=323)$

\begin{tabular}{|c|c|c|c|c|c|}
\hline Background of mentors & $n$ & $\%$ & Background of mentors & $n$ & $\%$ \\
\hline Gender & & & Experience living and/or working abroad & & \\
\hline Women & 277 & 86.0 & Yes & 99 & 30.7 \\
\hline Men & 45 & 14.0 & No & 224 & 69.3 \\
\hline Age & & & Frequency of mentoring exchange students & & \\
\hline Under 35 years & 105 & 32.5 & At least monthly & 4 & 1.2 \\
\hline $36-55$ years & 174 & 53.9 & Yearly & 70 & 21.7 \\
\hline Over 56 years & 44 & 13.6 & Less frequently & 147 & 45.5 \\
\hline Home country & & & No experience & 102 & 31.6 \\
\hline Finland & 316 & 97.8 & Frequency of mentoring ELTDP* students & & \\
\hline Other & 7 & 2.2 & At least monthly & 5 & 1.5 \\
\hline Native language & & & Yearly & 58 & 18.0 \\
\hline Finnish & 312 & 96.5 & Less frequently & 156 & 48.3 \\
\hline English & 3 & 0.9 & No experience & 104 & 32.2 \\
\hline Swedish & 4 & 1.2 & Frequency of mentoring CALD students in a FDP* & & \\
\hline German & 1 & 0.3 & At least monthly & 8 & 2.5 \\
\hline Russian & 2 & 0.6 & Yearly & 72 & 22.3 \\
\hline Other & 1 & 0.3 & Less frequently & 119 & 36.8 \\
\hline Educational background & & & No experience & 124 & 38.4 \\
\hline University of Applied Sciences & 297 & 92.0 & English language proficiency & & \\
\hline University & 19 & 5.8 & No competence & 2 & 0.6 \\
\hline Other & 7 & 2.2 & Basic & 59 & 18.3 \\
\hline Work experience & & & Advanced & 260 & 80.5 \\
\hline $0-5$ years & 53 & 16.4 & Native & 2 & 0.6 \\
\hline $6-10$ years & 64 & 19.8 & Sufficient knowledge of student's cultural background & & \\
\hline $11-20$ years & 109 & 33.7 & Agree & 153 & 47.4 \\
\hline $21-30$ years & 64 & 19.8 & Disagree & 170 & 52.6 \\
\hline More than 31 years & 33 & 10.2 & Spend time discussing cultural differences with students & & \\
\hline Current job title & & & Agree & 250 & 77.4 \\
\hline Registered Nurse & 316 & 97.8 & Disagree & 73 & 22.6 \\
\hline Nurse management & 6 & 1.9 & Ensure that CALD and native students work together & & \\
\hline Nurse expert & 1 & 0.3 & Agree & 249 & 77.1 \\
\hline Current work unit & 6 & 0.3 & Disagree & 74 & 22.9 \\
\hline Outpatient clinic & 112 & 34.7 & Require Finnish or Swedish language skills from students & & \\
\hline Inpatient unit & 173 & 53.6 & Agree & 160 & 50.5 \\
\hline Inpatient and outpatient unit & 25 & 7.7 & Disagree & 163 & 49.5 \\
\hline Other & 13 & 4.0 & $\begin{array}{l}\text { Need more support from colleagues in mentoring } \\
\text { students }\end{array}$ & & \\
\hline Mentor students as & & & Agree & 159 & 49.2 \\
\hline Mentor responsible for students & 45 & 13.9 & Disagree & 164 & 50.8 \\
\hline Named mentor & 130 & 40.2 & Have received additional support from colleagues & & \\
\hline Mentor & 140 & 43.3 & Agree & 246 & 76.2 \\
\hline Other & 8 & 2.5 & Disagree & 77 & 23.8 \\
\hline Mentored students last & & & Rating of overall mentoring competence & & \\
\hline Last week & 123 & 38.1 & Poor & 38 & 11.7 \\
\hline Last month & 109 & 33.7 & Good & 235 & 72.8 \\
\hline Last year & 85 & 26.4 & Excellent & 50 & 15.5 \\
\hline Over a year ago & 6 & 1.9 & & & \\
\hline \multicolumn{6}{|l|}{ Participated in mentoring training } \\
\hline Yes & 136 & 42.1 & & & \\
\hline No & 187 & 57.9 & & & \\
\hline
\end{tabular}

\footnotetext{
Note. ${ }^{*}$ ELTDP $=$ English language-taught degree program; FDP = Finnish degree program; CALD = culturally and linguistically diverse
} 
Table 3. MCI sub-dimensions $(n=323)$

\begin{tabular}{|c|c|c|c|c|}
\hline & Mean $\left(\mathrm{SD}^{*}\right)$ & Skewness & Kurtosis & Alpha \\
\hline \multicolumn{5}{|l|}{$\begin{array}{l}\text { MCI instrument 9-factor model } \\
\text { Sub-dimensions (total number of items) }\end{array}$} \\
\hline Mentor characteristics (7) & $3.57(0.40)$ & -0.61 & -0.72 & 0.87 \\
\hline Identifying student's level of competence (4) & $3.68(0.44)$ & -2.03 & 6.96 & 0.83 \\
\hline Motivation of the mentor (5) & $3.42(0.54)$ & -1.30 & 2.21 & 0.86 \\
\hline Motivating the student (4) & $3.49(0.46)$ & -0.90 & 1.07 & 0.77 \\
\hline Supporting the learning process of the student (7) & $3.51(0.37)$ & -0.47 & -0.69 & 0.79 \\
\hline Goal orientation in mentoring $(8)$ & $3.45(0.51)$ & -1.13 & 1.21 & 0.90 \\
\hline Reflection during mentoring (6) & $3.72(0.37)$ & -1.17 & 0.13 & 0.89 \\
\hline Student-centered feedback and evaluation (9) & $3.18(0.56)$ & -0.74 & 1.14 & 0.92 \\
\hline Constructive feedback and evaluation (5) & $3.48(0.42)$ & -0.45 & -0.68 & 0.75 \\
\hline
\end{tabular}

Note. *SD: standard deviation 
Table 4. Background factors that influence the mentoring of CALD nursing students based on logistic regression analysis $(n=323)$

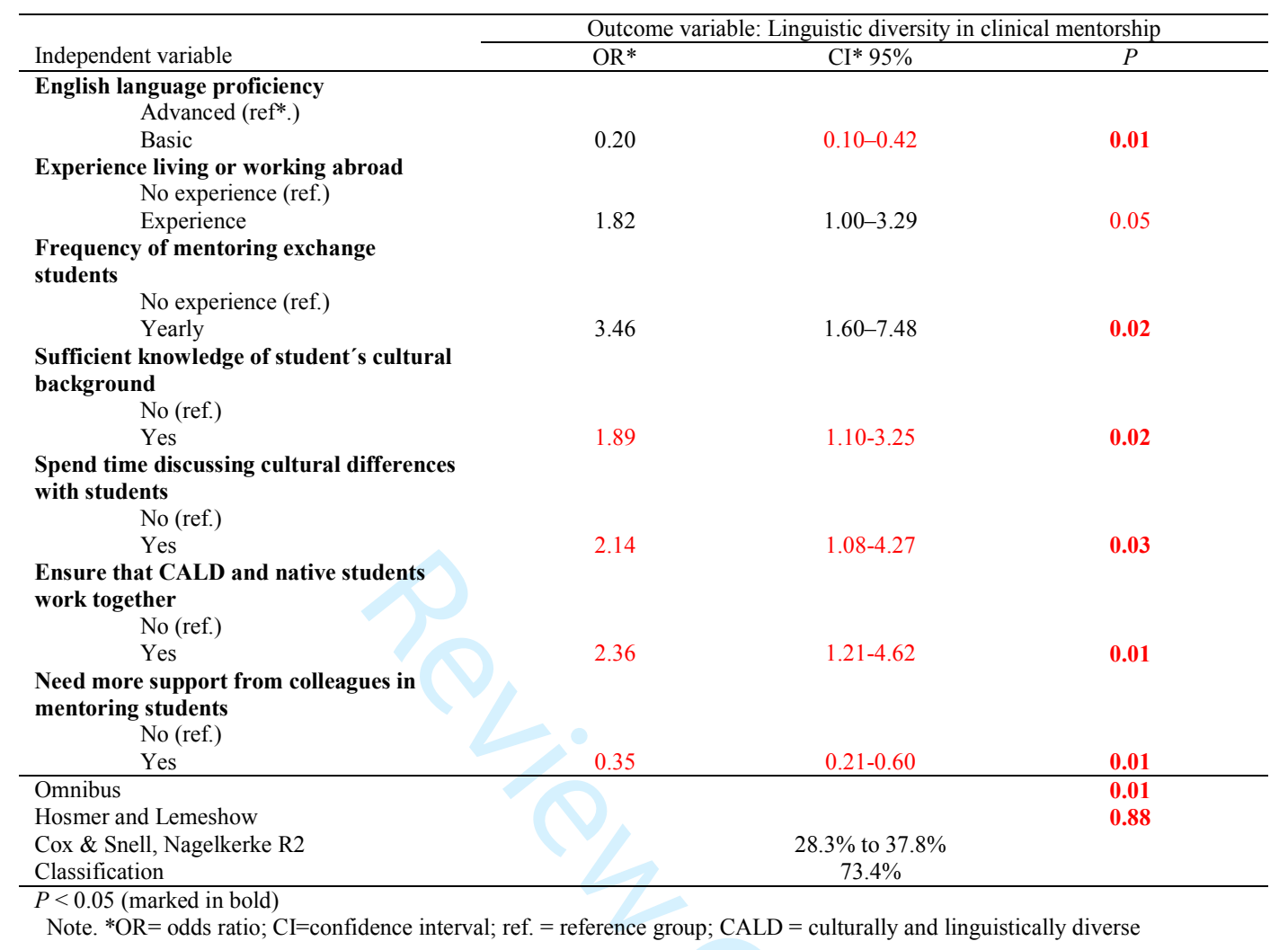


Dear Editor and Reviewers,

Thank you for your revisions and the helpful feedback you have provided to improve the manuscript. We feel honored to be given a possibility to make the revisions for the possible publication at your journal. Please find comments below to each point raised by the reviewers. We hope that our answers and corrections will meet your expectations and provide the answers you are looking for.

Please find our corrections in the manuscript marked in red.

EDITOR'S COMMENTS: Please will you address the reviewers' comments below, along with the following. Author Response to Comments:

Thank you for the clear instructions. We have considered every comment and completed the required revisions by adding author response to the comments.

1. Introduction/Background. Benchmark the international figures for migrant student nurses. State the international relevance/context of the issue studied. What is the size/proportion of students per total in various countries? In Finland, how many are migrant/international students?

We agree that the introduction/background section could benefit greatly from the benchmarking of international figures for migrant student nurses. At the moment, it appears that the data on such figures is lacking in Finland and in many other countries for the amount international/migrant nursing students. However, the figures for practicing nurses is much more readily available. We added a brief presentation of the growing diversity in the nursing workforce in Finland, with the percentages of practicing nurses with foreign backgrounds. Although there is a relatively small proportion of practicing nurses of foreign origin in Finland, these numbers have steadily increased, which brings forth implications for new, effective strategies to train these nurses along with nursing students entering the field.

The OECD (https://stats.oecd.org/Index.aspx?DataSetCode=RFOREIGN ) presents the figures of foreign/international students enrolled in, for example, total tertiary education in several countries. We believe that these numbers have limited relevancy to nursing education as we are not able to make a clear connection to what the numbers mean in this context. We strongly believe that gathering data on the figures for migrant nursing students is a really good idea for future development.

2. There is a very low response rate and reliance on one sample source. Making claims based on these findings is biased. Need to pick up on that in the Limitations.

Author Response to Comments:

Thank you for the important remark. We recognize the problem of the low response rate and further address the issue in the chapter of limitations.

3. A sub-section Reliability and Validity is required after Data analysis. How were R\&V assured for both data collection and data analysis.

Author Response to Comments:

The total sub-chapter has been added into the manuscript after sub-chapter of data analysis.

4. Discussion. How does this study advance existing nursing knowledge for policy, practice and research? Make explicit. What are the implications? 


\section{Author Response to Comments:}

Thank you for this important comment. We have added the following paragraphs and statements concerning policy, practice and research in Discussion chapter: second paragraph first sentence, the whole fourth paragraph excluding two first sentences, last paragraph last two sentences; in Conclusion chapter, nearly all chapter was rewritten.

5. Language screening and test requirements of students prior to acceptance on nurse education/training should be discussed and borne in mind when interpreting the results. What requirements are in place prior to admission to the course/s?

\section{Author Response to Comments:}

Language screening and test requirements of CALD students prior to acceptance to nursing education was discussed in the discussion in a fourth paragraph. An evaluation regarding language screening prior to entry to nursing programmes was presented, and comparison was made to the results of this study.

Reviewer: 1

Comments to the Author

6. The manuscript reports the results of a cross sectional survey of mentors of culturally and linguistically diverse (CALD) nursing students. In addition, although not stated as a formal objective, data were used to obtain evidence of validity and reliability of an instrument called CALD in Mentoring scale (CALD-Ms).

\section{Author Response to Comments:}

Thank you for this valuable comment.

7. The methodology for continued instrument development is appropriate. The authors need to add the names of the test statistics listed on p.8, lines 22-25.

Author Response to Comments:

Our apologies for not making the methods clear. We have specified the methods according to your instructions.

8. Study sample was obtained using stratified sampling, but strata and sampling technique were not described. Further, apparently neither strata nor sampling weights were used in the analysis. This would be appropriate if the goal was to simply test the associations among variables and not produce estimates of the effects in the population (e.g., percentages of women mentors or their educational backgrounds in the population). The statement of the first objective is not clear in this respect. If producing population estimates was not the goal, it would still be helpful to know what the strata and sampling plan were.

\section{Author Response to Comments:}

We have described the stratified sampling technique in the chapter The study, participants, in the first paragraph according to your instructions. We have used stratified groups as registered nurses representing each hospital of all five university hospitals in Finland (see table below). Our research goal was not compare the outcomes between the five hospitals, but rather get the total picture of the situation in Finland.

The stratified sampling technique was used by counting the total amount of registered nurses ( $\mathrm{N}=13$ 342) working in all five university hospitals in Finland. The information was provided by the administration from each university hospital. The stratified groups were divided into five groups according to the proportional amount of registered nurses 
representing the five different hospitals. The participants were chosen randomly in alphabetic order from each of the five groups with a total of 3355 nurses receiving an invitation to participate in the study.

\begin{tabular}{ccc}
\hline Hospitals (abbreviations) & Registered nurses & Sample size \\
\hline Hyks & 6000 & 1500 \\
Kys & 1572 & 435 \\
Oys & 2071 & 500 \\
Tays & 1738 & 420 \\
Tyks & 1961 & 500 \\
Total & $\mathbf{1 3 3 4 2}$ & $\mathbf{3 3 5 5}$ \\
\hline
\end{tabular}

9. Please change "internal consistency" to "internal consistency reliability" on p.7 and elsewhere in the manuscript when referencing Cronbach's alpha.

\section{Author Response to Comments:}

We have changed this according to your instructions. We have additionally moved reliability testing into the sub-chapter validity and reliability of the study under the main chapter The study.

10. Please provide a rationale for a cut-off of 2.50 in creating the binary response variable for logistic regression. The results of logistic regression modeling are well presented, with $95 \%$ confidence accompanying the odds ratio estimates. The p-values need to be rounded to 2 decimal places.

Author Response to Comments:

The four-level Likert scale was minimized into dichotomous response categories by dividing the scale into half according to the meaning of each scales item: (0) poor competence (12.49) and (1) good competence (2.50-4). Since the 1-2 include the meaning of disagreeing, and 3 and 4 agreeing, we chose the 2.50 to be a cut-off for the transforming the variables into dichotomous variables. We have added a sentence to explain this into the manuscript. The $\mathrm{p}$-values were rounded to 2 decimal as you have instructed.

11. The approach to building multivariable logistic regression model is p-value-based and not appropriate. When there are associations among potential explanatory variables, stepwise variable selection has multiple problems well documented in the statistical literature (see, for example, http://biostat.mc.vanderbilt.edu/wiki/Main/ManuscriptChecklist ). The use of subject matter expertise combined with careful backward elimination may be a better approach, with insignificant predictors removed only when they do not change the estimates of other effects in the model.

Author Response to Comments:

Thank you for your valuable instructions and sharing of the sources. We have performed regression analysis again by using of backward elimination with theoretical guidance on the subject matter. Additionally, we have a manually build, no-stepwise model, by testing each individual independent variable with the outcome and testing out various combinations of variables to find best fitting model. The independent variables remained the same, but the odds ratios and confidence intervals changed in some variables. The changes are marked in red and were adjusted in the text and table 4 accordingly.

Reviewer: 2 
Comments to the Author

A most interesting manuscript and topic! I congratulate the author(s) for their scholarly research and encourage them in their pursuit of publication. I provide the following feedback with respect and appreciation!

Author Response to Comments:

Thank you very much for your encouraging comments and constructive feedback. We appreciate it very much.

12. This manuscript is focused on mentoring in the education of nurses; the focus is the selfevaluation of mentors. Not what the students think, not what the patient thinks, rather what the mentors think of their mentoring. Periodically during this manuscript it would seem wise to (re)mention that this paper is about mentors self-perceptions....

Author Response to Comments:

Thank you for this important comment. We made sure that there is enough emphasis on selfperception throughout all manuscript.

13. Background Healthcare education is faced by several challenges (healthcare education is confronted by...)

Author Response to Comments:

This was corrected.

\section{Results}

Mentors with experience mentoring nursing students with diverse backgrounds Mentors with experience mentoring nursing students from diverse...

Author Response to Comments:

This was corrected.

15. What are the key findings?

- Mentors rated their competence in mentoring culturally and linguistically diverse nursing students positively with the exception of competence in linguistic diversity in mentoring.

- $\quad$ Mentors' competence in linguistic diversity was affected, among other matters, by English language proficiency, experience living or working abroad and frequency of mentoring exchange students.

- There is a need for further development of mentors' competence in cultural diversity, especially in reducing stereotypes and in increasing reflection on cultural differences with culturally diverse students.

The first dot point talks about 'linguistic diversity in mentoring' as the exception requiring further investigation. The third dot point talks about 'cultural diversity' as needing investigation. As written it sounds contradictory...?

\section{Author Response to Comments:}

Thank you for bringing this issue to our attention. We changed the wording of the third dot point to state that despite mentors' high overall evaluation of competence in cultural diversity, there is a need to address stereotypes and the lack of knowledge of student's background. The reduction of stereotypes was an item in the sub dimension, which received the lowest evaluation. We changed the wording regarding the background factor of mentors' knowledge of students' cultural background. 


\section{INTRODUCTION}

Healthcare systems are characterized by ever increasing diversity as a result of trends in both nurse migration and international migration in general (Bhopal 2014)

'...by ever increasing workforce diversity...' (for clarity, please)

Author Response to Comments:

We agree that it is more clear to state ever increasing workforce diversity, and have made this change.

17. The concept of 'culturally and linguistically diverse' (CALD) is used in this study to refer to nursing students whose background differ from the mainstream culture and language (Robinson \& Clardy 2011).

'...differs...'

Author Response to Comments:

This was corrected.

18. In the context of this study, having a CALD background means that the student has a native language, cultural background or heritage differing from that of the country he/she lives in. '...works in.'

Author Response to Comments:

Thank you for bringing this to our attention. In the context of this study, a CALD background refers to nursing students. For this reason, we changed the wording to "studies in".

19. In addition, several challenges exist when language barriers hinder communication between the mentor and student, however, in positive clinical learning environments the student's lack of language proficiency does not hinder learning (Myhre 2011, Pitkäjärvi et al. 2012b), different languages are used flexibly in different situation (Mattila et al. 2010) and the student receives the necessary support from the mentor for enabling communication (Authors names blinded).

Sentence too long.

Author Response to Comments:

This sentence was formed into two sentences.

20. THE STUDY

Aim

The aim of the study was to describe mentors' competence in mentoring culturally and linguistically diverse nursing students during clinical placement and identify the factors that affect mentoring. The research questions were (1) What kind of competencies do mentors have in mentoring CALD nursing students? and (2) Which factors affect the mentoring of CALD nursing students in the clinical learning environment?

The research questions should include clarity in the fact that this is what mentors selfassess? What mentors think...?

Author Response to Comments:

We have added self-perception emphasis into the second research question.

21. The data sample used in this study consisted of all respondents to the questionnaire who had experience in mentoring CALD nursing students (56.1\%).

Was the survey seeking other, unrelated data or can it be assumed the questionnaire was seeking general information about mentoring. If 576 responded to the survey and $56 \%$ were 
'eligible' do we assume some 288 responses? The majority of mentors (88.3\%) rated their overall mentoring competence as 'good' or excellent. Wow!

Author Response to Comments:

The total number of mentors with experience in mentoring CALD nursing students was $\mathrm{n}=$ 323. We have clarified the total number in the manuscript. The rest of the data is under analysis process and will be reported in another study.

22. Ministry of Education and Culture. (2012) Education and Research 2011-2016. A development plan. Available at: http://www.minedu.fi/OPM/Julkaisut/2012/Kehittamissuunnitelma.html?lang=fi\&extra_loca le=en (accessed 6 January 2017).

Munro B.H. 2005. Statistical Methods for Health Care Research. 5th edn. Lippincott Williams \& Wilkins, Philadelphia. Parenthesis for year of full-stop? Author Response to Comments: This was corrected according to the Journal of Advanced Nursing instructions. Our apologies for the inconsistency.

23. Williams B, Brown T \& Onsman A (2012) Exploratory factor analysis: A five-step guide for novices. Australasian Journal of Paramedicine 8(3), 1-12.

Yong A.G. \& Pearce S. (2013). A beginner guide to factor analysis: Focusing on exploratory factor analysis. Tutorials in Quantitative Methods for Psychology 9(2), 79-94. Full stop after parenthesis or not? Author Response to Comments:

This was corrected according to the Journal of Advanced Nursing instructions.

Reviewer: 3

Comments to the Author This paper was well written and contributes to new knowledge with the new measure.

Author Response to Comments:

Thank you for your valuable comments.

Only two minor corrections:

24. Page 14. No need for the full stop before the (Myhre 2011) reference. Author Response to Comments: This was corrected according to the Journal of Advanced Nursing instructions. Our apologies for inconsistency.

25. Page 17. The phrasing of the last sentence in the last paragraph on page 17 could be improved. ('Was able to be performed' does not sound great in this context - consider rephrasing the sentence).

Author Response to Comments: Thank you for this suggestion. The sentence was modified accordingly. 
STROBE Statement — checklist of items that should be included in reports of observational studies

\begin{tabular}{|c|c|c|}
\hline & $\begin{array}{l}\text { Item No } \\
\text { X-indicate } \\
\text { authors } \\
\text { answer } \\
\end{array}$ & $\begin{array}{l}\text { Recommendation } \\
\begin{array}{c}\text { Authors marked checklist in bold, which were applicable to our study } \\
\text { and all added explanations were underlined }\end{array} \\
\end{array}$ \\
\hline \multirow[t]{2}{*}{ Title and abstract } & \multirow[t]{2}{*}{$1 \mathrm{X}$} & $\begin{array}{l}\text { (a) Indicate the study's design with a commonly used term in the title or } \\
\text { the abstract }\end{array}$ \\
\hline & & $\begin{array}{l}\text { (b) Provide in the abstract an informative and balanced summary of } \\
\text { what was done and what was found }\end{array}$ \\
\hline \multicolumn{3}{|l|}{ Introduction } \\
\hline Background/rationale & $2 \mathrm{X}$ & $\begin{array}{l}\text { Explain the scientific background and rationale for the investigation } \\
\text { being reported }\end{array}$ \\
\hline Objectives & $3 X$ & State specific objectives, including any prespecified hypotheses \\
\hline \multicolumn{3}{|l|}{ Methods } \\
\hline Study design & $4 X$ & Present key elements of study design early in the paper \\
\hline Setting & $5 X$ & $\begin{array}{l}\text { Describe the setting, locations, and relevant dates, including periods of } \\
\text { recruitment, exposure, follow-up, and data collection }\end{array}$ \\
\hline \multirow[t]{2}{*}{ Participants } & 6 & $\begin{array}{l}\text { (a) Cohort study-Give the eligibility criteria, and the sources and methods } \\
\text { of selection of participants. Describe methods of follow-up } \\
\text { Case-control study - Give the eligibility criteria, and the sources and } \\
\text { methods of case ascertainment and control selection. Give the rationale for } \\
\text { the choice of cases and controls }\end{array}$ \\
\hline & $\mathrm{X}$ & $\begin{array}{l}\text { Cross-sectional study - Give the eligibility criteria, and the sources and } \\
\text { methods of selection of participants }\end{array}$ \\
\hline
\end{tabular}

(b) Cohort study - For matched studies, give matching criteria and number of exposed and unexposed

Case-control study - For matched studies, give matching criteria and the number of controls per case

\begin{tabular}{lcl}
\hline Variables & $7 \mathrm{X}$ & $\begin{array}{l}\text { Clearly define all outcomes, exposures, predictors, potential confounders, } \\
\text { and effect modifiers. Give diagnostic criteria, if applicable }\end{array}$ \\
\hline $\begin{array}{l}\text { Data sources/ } \\
\text { measurement }\end{array}$ & $8^{*} \mathrm{X}$ & $\begin{array}{l}\text { For each variable (sum-variable) of interest, give sources of data and } \\
\text { details of methods of assessment (measurement). Describe comparability } \\
\text { of assessment methods if there is more than one group }\end{array}$ \\
\hline Bias & $9 \mathrm{X}$ & Describe any efforts to address potential sources of bias \\
\hline Study size & $10 \mathrm{X}$ & Explain how the study size was arrived at \\
\hline Quantitative variables & $11 \mathrm{X}$ & $\begin{array}{l}\text { Explain how quantitative variables were handled in the analyses. If } \\
\text { applicable, describe which groupings were chosen and why }\end{array}$ \\
\hline Statistical methods & $12 \mathrm{X}$ & $\begin{array}{l}\text { (a) Describe all statistical methods, including those used to control for } \\
\text { confounding }\end{array}$ \\
\hline
\end{tabular}

(b) Describe any methods used to examine subgroups and interactions

(c) Explain how missing data were addressed

(d) Cohort study - If applicable, explain how loss to follow-up was addressed

Case-control study —If applicable, explain how matching of cases and controls was addressed

Cross-sectional study—If applicable, describe analytical methods taking 
account of sampling strategy

Continued on next page

\section{(e) Describe any sensitivity analyses}


Results

\begin{tabular}{lcl}
\hline Participants & $13^{*}$ & $\begin{array}{l}\text { (a) Report numbers of individuals at each stage of study-eg numbers potentially } \\
\mathrm{X}\end{array}$ \\
& & $\begin{array}{l}\text { eligible, examined for eligibility, confirmed eligible, included in the study, completing } \\
\text { follow-up, and analysed }\end{array}$ \\
\hline & $14 * \mathrm{X}$ (b) Give reasons for non-participation at each stage \\
\hline $\begin{array}{l}\text { (c) Consider use of a flow diagram } \\
\text { Descriptive }\end{array}$ & $\begin{array}{l}\text { (a) Give characteristics of study participants (eg demographic, clinical, social) and } \\
\text { information on exposures and potential confounders }\end{array}$ \\
\hline data &
\end{tabular}

(b) Indicate number of participants with missing data for each sum-variables of interest

(c) Cohort study-Summarise follow-up time (eg, average and total amount)

\begin{tabular}{|c|c|c|}
\hline \multirow[t]{3}{*}{ Outcome data } & \multirow[t]{3}{*}{$15 * X$} & Cohort study_Report numbers of outcome events or summary measures over time \\
\hline & & $\begin{array}{l}\text { Case-control study-Report numbers in each exposure category, or summary measures of } \\
\text { exposure }\end{array}$ \\
\hline & & $\begin{array}{l}\text { Cross-sectional study-Report numbers of outcome events or summary measures (of } \\
\text { factory analysis and sum-variables) }\end{array}$ \\
\hline \multirow[t]{3}{*}{ Main results } & \multirow[t]{3}{*}{$16 \mathrm{X}$} & $\begin{array}{l}\text { (a) Give unadjusted estimates and, if applicable, confounder-adjusted estimates and their } \\
\text { precision (eg, } 95 \% \text { confidence interval). Make clear which confounders were adjusted for } \\
\text { and why they were included }\end{array}$ \\
\hline & & (b) Report category boundaries when continuous variables were categorized \\
\hline & & $\begin{array}{l}\text { (c) If relevant, consider translating estimates of relative risk into absolute risk for a } \\
\text { meaningful time period }\end{array}$ \\
\hline Other analyses & $17 \mathrm{X}$ & $\begin{array}{l}\text { Report other analyses done }- \text { eg analyses of subgroups and interactions, and sensitivity } \\
\text { analyses }\end{array}$ \\
\hline \multicolumn{3}{|l|}{ Discussion } \\
\hline Key results & $18 \mathrm{X}$ & Summarise key results with reference to study objectives \\
\hline Limitations & $19 X$ & $\begin{array}{l}\text { Discuss limitations of the study, taking into account sources of potential bias or } \\
\text { imprecision. Discuss both direction and magnitude of any potential bias }\end{array}$ \\
\hline Interpretation & $20 \mathrm{X}$ & $\begin{array}{l}\text { Give a cautious overall interpretation of results considering objectives, limitations, } \\
\text { multiplicity of analyses, results from similar studies, and other relevant evidence }\end{array}$ \\
\hline Generalisability & $21 \mathrm{X}$ & Discuss the generalisability (external validity) of the study results \\
\hline \multicolumn{3}{|c|}{ Other information } \\
\hline Funding & $22 X$ & $\begin{array}{l}\text { Give the source of funding and the role of the funders for the present study and, if } \\
\text { applicable, for the original study on which the present article is based }\end{array}$ \\
\hline
\end{tabular}

*Give information separately for cases and controls in case-control studies and, if applicable, for exposed and unexposed groups in cohort and cross-sectional studies.

Note: An Explanation and Elaboration article discusses each checklist item and gives methodological background and published examples of transparent reporting. The STROBE checklist is best used in conjunction with this article (freely available on the Web sites of PLoS Medicine at http://www.plosmedicine.org/, Annals of Internal Medicine at http://www.annals.org/, and Epidemiology at http://www.epidem.com/). Information on the STROBE Initiative is available at www.strobe-statement.org. 